Zhao, P., et al., 2021, Early Neoproterozoic (870-820 Ma) amalgamation of the Tarim craton (northwestern

Supplementary Material for:

\title{
Early Neoproterozoic (870-820 Ma) amalgamation of the Tarim Craton and the final assembly of Rodinia
}

\author{
Pan Zhao, Jinyou He, Chenglong Deng, Yan Chen, and Ross Mitchell
}

\section{This file contains:}

Supplementary text

Supplementary figures (Fig. S1, S2)

Supplementary tables (Table S1, S2, S3)

Supplementary references

\section{SUPPLEMENTARY TEXT}

\section{Paleomagnetic sampling and laboratory procedure}

The paleomagnetic samples were collected from the newly identified Qigelekekuotan volcanic sequence (QV; Fig. S2). In total, 21 sites (194 cores) were drilled using a portable gasoline drilling machine. Cores were orientated in the field by both magnetic and solar compasses.

In the laboratory, core samples $(2.5 \mathrm{~cm}$ in diameter $)$ were cut into standard cylinder specimens with $2.2 \mathrm{~cm}$ in height. Systematic rock magnetic investigations were performed to determine the magnetic carrier(s). High-temperature thermomagnetic experiments $(\kappa-T)$ were performed using a MFK-1 Kappa-bridge coupled with a CS3 furnace. Acquisition and back-field demagnetization of isothermal remanent magnetization (IRM) and magnetic hysteresis loops were measured using a MicroMag 3900 Alternating Gradient Magnetometer with an applied filed in the range of $\pm 1.5 \mathrm{~T}$. All these magnetic mineralogy investigations were conducted in the Paleomagnetism and Geochronology Laboratory (PGL) of the Institute of Geology and Geophysics, Chinese Academy of Sciences.

All standard specimens were subjected to stepwise demagnetization. Only thermal demagnetization technique was applied to remove the magnetic remanence progressively by 13-16 steps up to $685^{\circ} \mathrm{C}$, with temperature intervals of $20^{\circ} \mathrm{C}$ to $100^{\circ} \mathrm{C}$. A thermal demagnetizer (model TD-48SC of the ASC Scientific Company) was used for demagnetization, which is followed by magnetic remanence measurement with a $2 \mathrm{G}$ Enterprises three-axis SQUID Magnetometer (Model 755) installed in a magnetically shielded room with residual field of less than $200 \mathrm{nT}$ in the PGL.

Magnetic directions were analyzed by principal component analysis (Kirschvink, 1980). 
Mean remanence directions were computed by Fisher statistics by paleomagnetic software packages of Remasoft (designed by AGICO) and PaleoMac (Cogné, 2003).

\section{Rock magnetism}

Rock magnetic experiments Thermomagnetic $(\kappa-T)$ curves show irreversible heating and cooling behaviors (Figs. S2A-C). The heating curves show a significant decrease of the magnetic susceptibility values at $\sim 580^{\circ} \mathrm{C}$, and then a gradual decay until $700^{\circ} \mathrm{C}$ (Figs. S2A-C), indicating the existence of both magnetite and hematite as magnetic remanence carriers. The hysteresis loops of studied samples (Figs. S2D-F) show wasp-waisted shape with coercivity $(\mathrm{Hc}$ ) values of $48-90 \mathrm{mT}$ and $\mathrm{Mr} / \mathrm{Ms}$ ratios at $\sim 0.4$, suggesting that both soft (magnetite) and hard (hematite) magnetic minerals exist as magnetic carriers. IRM acquisition curves show a rapid increase of magnetization from 0-200 $\mathrm{mT}$, followed by a gradual increase of magnetization without saturation at $1.5 \mathrm{~T}$ (Figs. S2G-I), indicating the coexistence of magnetite and hematite. Unmixing of magnetic mineral components also reveals both soft and hard mineral components (Figs. S2J-L). From rock magmatic experiments, magnetite and hematite can be considered as the main magnetic remanence carriers in the studied volcanic samples.

Reference:

Cogné, J.P., 2003, A Macintosh ${ }^{\mathrm{TM}}$ application for treating paleomagnetic data and making plate reconstructions: Geochemistry, Geophysics, Geosystems, v. 4, p. 1007, doi:10.1029/2001GC000227.

Kirschvink, J.L., 1980, The least-squares line and plane and the analysis of paleomagnetic data: Geophys. J. R. Astron. Soc., v. 62, p. 699-718, doi:10.1111/j.1365-246X.1980.tb02601.X. 


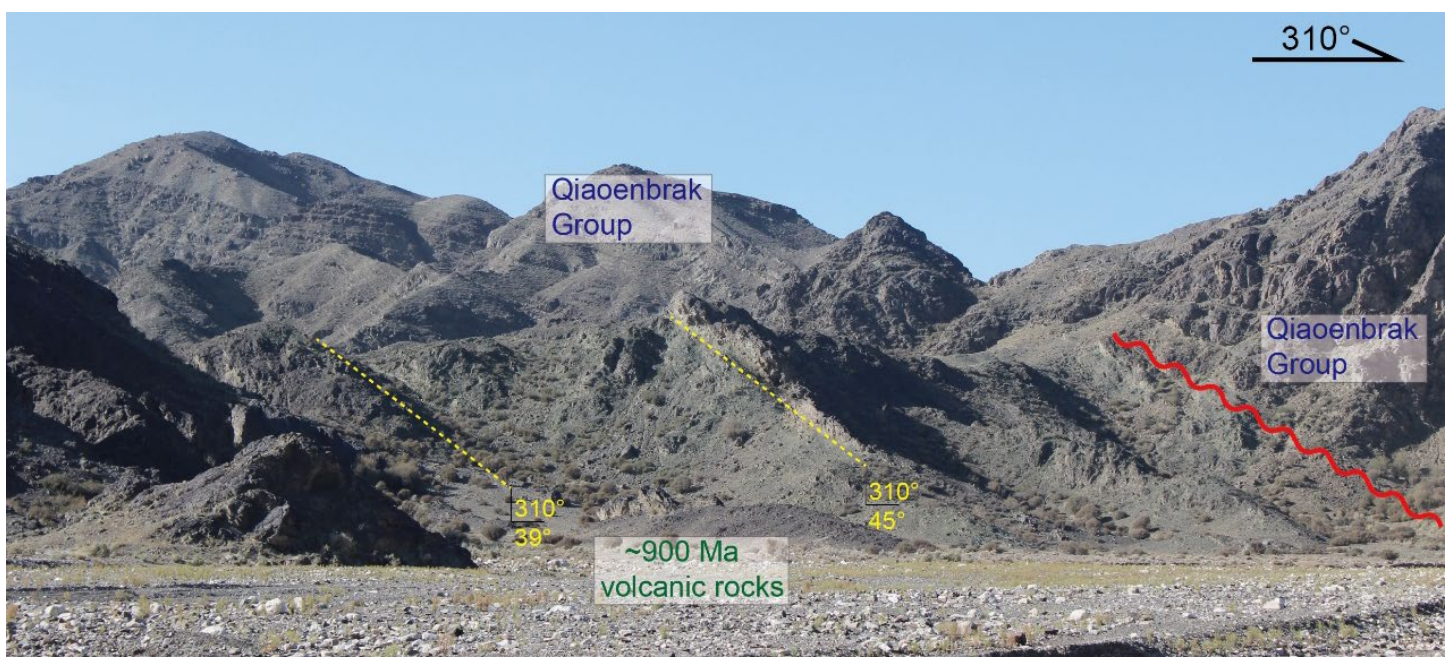

Fig. S1: Photo of field outcrop of the $\sim 900$ Ma Qigelekekuotan volcanic sequence showing dip and unconformable relationship with the Qiaoenbrak Group.
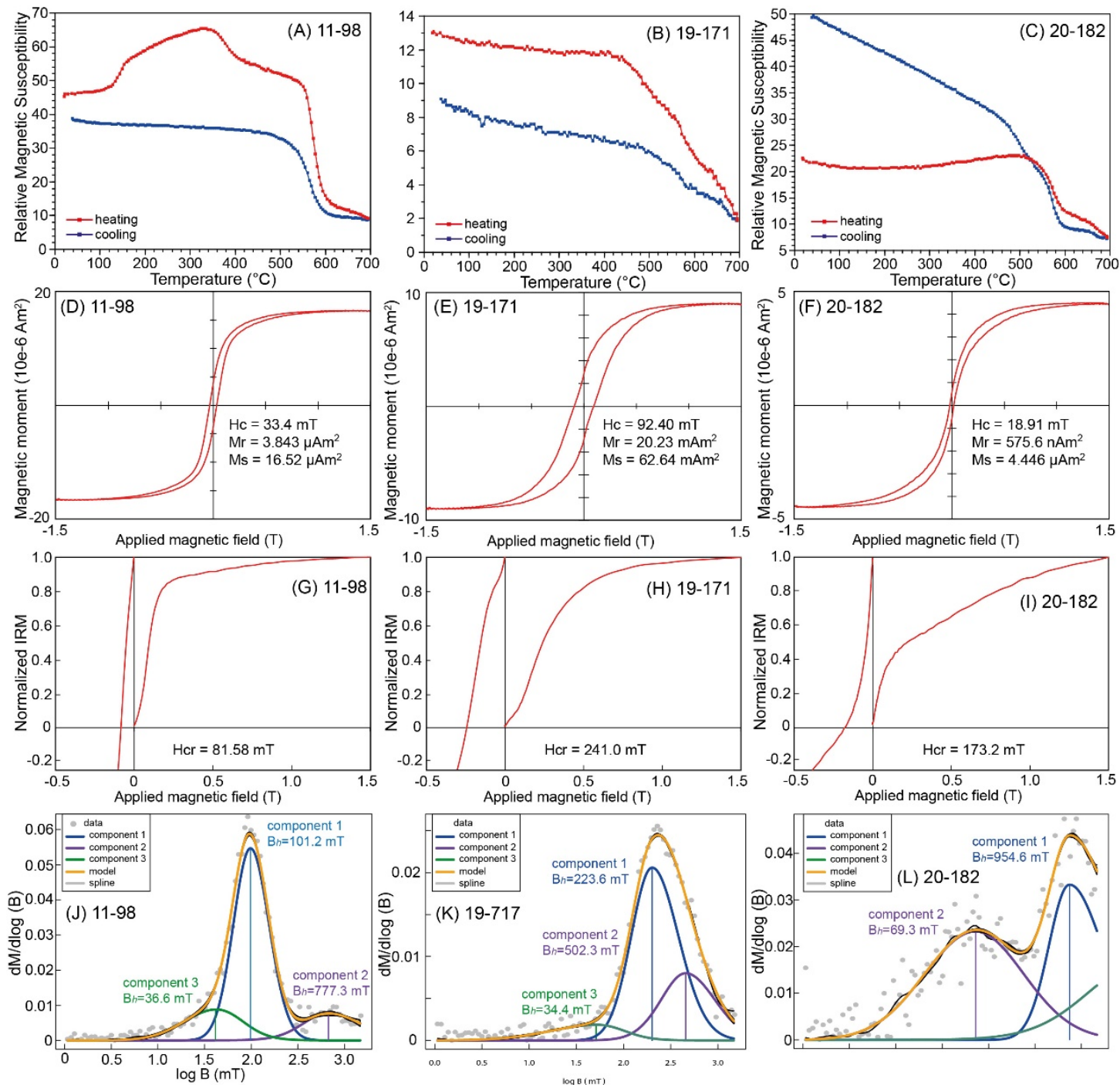

Fig. S2: Rock magnetic experiments. Thermomagnetic curves of magnetic susceptibility (A-C), magnetic hysteresis loops (D-F), isothermal magnetization acquisition and back-field demagnetization of saturation IRM (SIRM) acquisition curves (G-I), and unmixing of magnetic mineral components modeled with MAX UnMix (J-L) of selected samples. 


\section{SUPPLEMENTARY TABLES}

Table S1: Paleomagnetic sampling and measurement results of the $\sim 900$ Ma volcanic rocks from the Aksu region in northwestern Tarim craton

Table S2: Paleomagnetic poles for the Tarim craton from the Neoproterozoic to present.

Table S3: Compilation of 1000-850 Ma palaeomagnetic poles from main cratons of Rodinia. Abbreviations: Slat (Plat), latitude of sampling site (pole); Slong (Plong), longitude of sampling site (pole); A95, 95\% confidence limit; FT, fold test; CT, conglomerate test; $\mathrm{BT}$, backed test; $\mathrm{N}+\mathrm{R}$, existence of both normal and reversed polarities. 
Table S1. Paleomagnetic sampling and measurement results of the $\sim 900$ Ma volcanic rocks from the Aksu region in northwestern Tarim Craton

\begin{tabular}{|c|c|c|c|c|c|c|c|c|c|c|c|c|c|c|}
\hline Site & coordinate & rock type & $\begin{array}{c}\text { dip } \\
\text { dir./angle }\end{array}$ & $\mathbf{n}^{\prime} / \mathbf{n}$ & $\mathbf{N} / \mathbf{R}$ & Dg & Ig & Ds & Is & $\mathbf{k} / \mathbf{K}$ & $\alpha_{95} / \mathbf{A}_{95}$ & $s / S$ & $\begin{array}{l}\text { Plat. } \\
\left({ }^{\circ}\right)\end{array}$ & $\begin{array}{c}\text { Plong. } \\
\left({ }^{\circ} \mathbf{E}\right)\end{array}$ \\
\hline $01-03$ & $40^{\circ} 56^{\prime} 38^{\prime \prime} \mathrm{N}, 79^{\circ} 30^{\prime} 12^{\prime \prime} \mathrm{E}$ & feldspar-phenocryst-bearing andesite & $315 / 40$ & $6 / 24$ & $5 / 1$ & 182.6 & 34.9 & 219.7 & 52.6 & 41.2 & 10.6 & 13.6 & -7.4 & 46.9 \\
\hline 04 & $40^{\circ} 56^{\prime} 39^{\prime \prime} \mathrm{N}, 79^{\circ} 30^{\prime} 13^{\prime \prime} \mathrm{E}$ & feldspar-phenocryst-bearing andesite & $315 / 40$ & $7 / 8$ & $7 / 0$ & 197.7 & 45.1 & 243.3 & 48.7 & 70.9 & 7.2 & 25.5 & 1.6 & 28.5 \\
\hline 05 & $40^{\circ} 56^{\prime} 39^{\prime \prime} \mathrm{N}, 79^{\circ} 30^{\prime} 13^{\prime \prime} \mathrm{E}$ & feldspar-phenocryst-bearing andesite & $315 / 40$ & $0 / 6$ & $0 / 0$ & - & - & - & - & - & - & & & \\
\hline 06 & $40^{\circ} 56^{\prime} 39^{\prime \prime} \mathrm{N}, 79^{\circ} 30^{\prime} 14^{\prime \prime} \mathrm{E}$ & feldspar-phenocryst-bearing andesite & $315 / 40$ & $6 / 13$ & $6 / 0$ & 131.0 & 22.1 & 187.6 & 49.9 & 49.2 & 9.9 & 16.9 & -18.1 & 72.6 \\
\hline $07^{*}$ & $40^{\circ} 56^{\prime} 40^{\prime \prime} \mathrm{N}, 79^{\circ} 30^{\prime} 13^{\prime \prime} \mathrm{E}$ & feldspar-phenocryst-bearing andesite & $315 / 40$ & $7 / 7$ & $7 / 0$ & 253.7 & 52.9 & 279.3 & 25.1 & 24.1 & 12.5 & 60.7 & 15.6 & 353.6 \\
\hline 08 & $40^{\circ} 56^{\prime} 43^{\prime \prime} \mathrm{N}, 79^{\circ} 30^{\prime} 10^{\prime \prime} \mathrm{E}$ & green andesite & $310 / 45$ & $12 / 13$ & $12 / 0$ & 159.9 & 36.8 & 210.5 & 66.1 & 16.9 & 10.9 & 3.1 & 3.3 & 59.8 \\
\hline 09 & $40^{\circ} 56^{\prime} 44^{\prime \prime} \mathrm{N}, 79^{\circ} 30^{\prime} 10^{\prime \prime} \mathrm{E}$ & green andesite & $310 / 45$ & $7 / 7$ & $7 / 0$ & 135.8 & 24.5 & 144.9 & 68.9 & 57.4 & 8.0 & 23.6 & 8.1 & 100.3 \\
\hline 10 & $40^{\circ} 56^{\prime} 44^{\prime \prime} \mathrm{N}, 79^{\circ} 30^{\prime} 10^{\prime \prime} \mathrm{E}$ & green andesite & $310 / 45$ & $7 / 7$ & $7 / 0$ & 133.2 & 26.3 & 138.8 & 71.1 & 26.6 & 11.9 & 24.9 & 12.6 & 101.9 \\
\hline 11 & $40^{\circ} 56^{\prime} 43^{\prime \prime} \mathrm{N}, 79^{\circ} 30^{\prime} 09^{\prime \prime} \mathrm{E}$ & green andesite & $310 / 45$ & $9 / 9$ & $9 / 0$ & 149.8 & 33.5 & 189.9 & 71.0 & 56.3 & 6.9 & 9.1 & 6.7 & 73.9 \\
\hline 12 & $40^{\circ} 56^{\prime} 43^{\prime \prime N}, 79^{\circ} 30^{\prime} 09^{\prime \prime} \mathrm{E}$ & green andesite & $310 / 45$ & $8 / 8$ & $8 / 0$ & 149.2 & 21.1 & 169.7 & 61.3 & 34.2 & 9.6 & 16.3 & -6.2 & 87.1 \\
\hline 13 & $40^{\circ} 56^{\prime} 44^{\prime \prime N}, 79^{\circ} 30^{\prime} 14^{\prime \prime} \mathrm{E}$ & green andesite & $310 / 39$ & $6 / 9$ & $6 / 0$ & 143.0 & 45.0 & 191.1 & 79.5 & 61.6 & 8.6 & 16.0 & 20.9 & 75.4 \\
\hline 14 & $40^{\circ} 56^{\prime} 09^{\prime \prime} \mathrm{N}, 79^{\circ} 30^{\prime} 43^{\prime \prime} \mathrm{E}$ & purple andesite & $265 / 26$ & $9 / 9$ & $9 / 0$ & 181.5 & 64.7 & 221.0 & 52.4 & 35.9 & 8.7 & 14.2 & -7.0 & 45.8 \\
\hline 15 & $40^{\circ} 56^{\prime} 09^{\prime \prime} \mathrm{N}, 79^{\circ} 30^{\prime} 43^{\prime \prime} \mathrm{E}$ & purple andesite & $265 / 26$ & $8 / 8$ & $8 / 0$ & 168.3 & 69.5 & 222.1 & 59.3 & 235.0 & 3.6 & 9.3 & -0.4 & 48.6 \\
\hline $16^{*}$ & $40^{\circ} 56^{\prime} 09^{\prime \prime} \mathrm{N}, 79^{\circ} 30^{\prime} 43^{\prime \prime} \mathrm{E}$ & purple andesite & $265 / 26$ & $7 / 10$ & $7 / 0$ & 322.4 & 68.8 & 292.6 & 48.8 & 25.5 & 12.2 & 46.5 & 35.2 & 0.5 \\
\hline $17^{*}$ & $40^{\circ} 56^{\prime} 09^{\prime \prime} \mathrm{N}, 79^{\circ} 30^{\prime} 43^{\prime \prime} \mathrm{E}$ & purple andesite & $265 / 26$ & $10 / 10$ & $10 / 0$ & 312.7 & 67.2 & 289.1 & 45.6 & 19.0 & 11.4 & 47.6 & 31.2 & 359.8 \\
\hline 18 & $40^{\circ} 56^{\prime} 09^{\prime \prime} \mathrm{N}, 79^{\circ} 30^{\prime} 44^{\prime \prime} \mathrm{E}$ & green andesite & $265 / 26$ & $4 / 5$ & $0 / 4$ & 13.3 & -68.5 & 50.8 & -51.8 & 56.8 & 12.3 & 18.0 & -3.0 & 38.6 \\
\hline 19 & $40^{\circ} 56^{\prime} 10^{\prime \prime} \mathrm{N}, 79^{\circ} 30^{\prime} 42^{\prime \prime} \mathrm{E}$ & purple andesite & $265 / 26$ & $3 / 9$ & $3 / 0$ & 171.3 & 74.7 & 232.1 & 61.0 & 87.2 & 13.3 & 12.7 & 5.4 & 43.4 \\
\hline 20 & $40^{\circ} 56^{\prime} 18^{\prime \prime N}, 79^{\circ} 30^{\prime} 32^{\prime \prime} \mathrm{E}$ & green tuff & $265 / 26$ & $10 / 10$ & $10 / 0$ & 155.0 & 62.5 & 204.6 & 60.0 & 50.7 & 6.9 & 4.0 & -5.2 & 61.1 \\
\hline 21 & $40^{\circ} 56^{\prime} 18^{\prime \prime} \mathrm{N}, 79^{\circ} 30^{\prime} 32^{\prime \prime} \mathrm{E}$ & green tuff & $265 / 26$ & $11 / 11$ & $11 / 0$ & 141.4 & 54.9 & 183.6 & 61.0 & 131.7 & 4.0 & 10.4 & -7.0 & 76.8 \\
\hline & Mean (15 sites) & & & & & 155.2 & 47.5 & & & 11.6 & 11.7 & & & \\
\hline & & & & & & & & 205.2 & 64.0 & 24.4 & 7.9 & & & \\
\hline \multicolumn{2}{|c|}{ VPGs mean } & & & & & & & & & 11.5 & 11.8 & 23.3 & 0.3 & 63.8 \\
\hline
\end{tabular}


Abbreviations: n'/n: numbers of accepted/measured samples; N/R: normal/reverse polarity; Dg, Ig, Ds, Is: declination (D) and inclination (I) in geographic (g) and tilt-corrected (s) coordinates; $\mathrm{k} / \mathrm{K}$ : precision parameter for site-mean direction and formation mean virtual geomagnetic pole (VGP); $\alpha_{95} / \mathrm{A}_{95}$ : radius of the $95 \%$ confidence circle for site-mean direction and formation mean VGP. Plong/Plat, longitude/latitude of VGP after tilt correction. $s / S$, angular deviations of site-mean directions and VGPs. *Sites were discarded for further discussion due to $>45^{\circ}$ angular deviations from mean direction. VGPs are calculated at reference site $40.9^{\circ} \mathrm{N}$ and $79.5^{\circ} \mathrm{E}$. Paleolatitude $=47.0^{\circ} \pm 11.8^{\circ} \mathrm{N}$ is calculated with VGPs mean.

McElhinny's (1964) fold test: $\mathrm{ks} / \mathrm{kg}=2.10>1.88$; indicate positive fold test at $95 \%$ confidence level.

McFadden's (1990) fold test: $\xi_{2}=9.819$ before, and $\xi_{2}=0.3884$ after tilt correction, critical value $\xi=4.510$ at $95 \%$ and $\xi=6.305$ at $99 \%$ confidence level; indicate positive fold test at $99 \%$ confidence level.

Enkin's (2003) DC fold test give positive feedback with DC Slope: $97.03 \% \pm 21.48 \%$ untilting.

Watson and Enkin's (1993) fold test give optimum degree of untilting at $96.0 \% \pm 12.2 \%$, indicating positive fold test.

The angular deviation of the 15 corresponding virtual geomagnetic poles (VGPs) $\left(S=23.3^{\circ}\right.$ at $\left.47^{\circ}\right)$ is similar with $S_{\lambda}=17.1^{\circ}\left(95 \%\right.$ confidence limits of $\left.14.3^{\circ}-20.4^{\circ}\right)$ at $43.1^{\circ} \mathrm{N}$ and $S_{\lambda}=20.7^{\circ}\left(95 \%\right.$ confidence limits of $\left.18.2^{\circ}-23.4^{\circ}\right)$ at $53.1^{\circ} \mathrm{N}$ (Johnson et al., 2008). The A95 value of the mean VGPs fall in the range of A 95 envelope defined by Deenen et al. $(2011)\left(\mathrm{A}_{95} \min =4.1^{\circ}<\mathrm{A}_{95}=11.8^{\circ}<\mathrm{A}_{95} \max =14.9^{\circ}\right)$. These results indicate that paleosecular variation has been averaged out. 
Table S2. Paleomagnetic poles for the Tarim Craton from the Neoproterozoic to present

\begin{tabular}{|c|c|c|c|c|c|c|c|c|c|}
\hline Age & $\mathbf{S}(\mathbf{N})$ & SLAT & SLON & PLAT & PLON & Dp/A95 & Dm & Reference & Comment \\
\hline \multicolumn{10}{|l|}{ Neoproterozoic } \\
\hline *NP1, 909-903 & 15 & 40.9 & 79.5 & -0.5 & 62.2 & 11.2 & & This study & $\mathrm{FT}, \mathrm{N}+\mathrm{R}$ \\
\hline *NP1, 900-870 & 11 & 37.0 & 77.3 & -23.5 & 37.0 & 11.3 & & Wen et al., 2018 & $\mathrm{CT}$ \\
\hline *NP2, 785-759 & 9 & 41.1 & 80.1 & 19.0 & 128.0 & 6.0 & 7.0 & Chen et al., 2004 & $\mathrm{~N}+\mathrm{R}$ \\
\hline *NP2, 750-725 & 6 & 41.6 & 86.5 & -17.7 & 14.2 & 3.0 & 6.0 & Huang et al., 2005 & \\
\hline *NP2, 730 & 14 & 40.9 & 79.5 & -6.3 & 17.5 & 9.1 & & Wen et al., 2013 & $\mathrm{CT}, \mathrm{N}+\mathrm{R}$ \\
\hline$* \mathrm{NP} 2,635$ & 6 & 41.5 & 87.8 & 27.6 & 140.4 & 8.8 & 11.1 & Zhao et al., 2014 & FT \\
\hline$* \mathrm{NP} 2,625$ & 24 & 41.0 & 80.0 & 19.1 & 149.7 & 9.3 & & Zhan et al., 2007 & $\mathrm{FT}, \mathrm{N}+\mathrm{R}$ \\
\hline *NP2, 625 & 36 & 41.0 & 79.3 & -21.1 & 87.4 & 7.0 & & Wen et al., 2017 & FT, N+R \\
\hline$* \mathrm{NP} 2,615$ & 8 & 41.5 & 87.8 & -4.9 & 146.7 & 3.0 & 5.2 & Zhao et al., 2014 & \\
\hline \multicolumn{10}{|c|}{ Early Ordovician } \\
\hline$* 480$ & 5 & 41.3 & 83.4 & -20.4 & 180.6 & 8.5 & 15.0 & Zhu et al., 1998 & \\
\hline \multicolumn{10}{|c|}{ Middle Ordovician } \\
\hline$* 460$ & 11 & 40.6 & 78.9 & -33.7 & 185.0 & 2.7 & 4.0 & Huang et al., 2019 & $\mathrm{~N}+\mathrm{R}$ \\
\hline \multicolumn{10}{|l|}{ Silurian } \\
\hline * 435 & 6 & 40.5 & 79.7 & 12.1 & 158.4 & 4.1 & 7.2 & Zhu et al., 1998 & \\
\hline \multicolumn{10}{|l|}{ Middle Silurian } \\
\hline$* 430$ & 10 & 40.5 & 78.7 & 19.1 & 172.9 & 5.5 & & Huang et al., 2019 & $\mathrm{FT}, \mathrm{N}+\mathrm{R}$ \\
\hline \multicolumn{10}{|c|}{ Early-middle Devonian } \\
\hline$* 400$ & 3 & 40.5 & 79.6 & 13.5 & 160.8 & 4.9 & & Fang et al, 1996 & \\
\hline \multicolumn{10}{|l|}{ Late Devonian } \\
\hline$* 370$ & 47 & 40.5 & 78.6 & 16.5 & 165.0 & 4.3 & & Li et al, 1990 & $\mathrm{FT}, \mathrm{N}+\mathrm{R}$ \\
\hline \multicolumn{10}{|c|}{ Late Carboniferous } \\
\hline $\mathrm{C}_{2}$ & 4 & 40.3 & 79.5 & 52.2 & 179.5 & 6.8 & 10.7 & Bai et al., 1987 & \\
\hline $\mathrm{C}_{2}$ & 3 & & & 48.7 & 175.7 & 3.0 & 4.8 & Fang et al., 1998 & \\
\hline *Ave, 310 & $2 / 2$ & & & 50.5 & 177.5 & 9.3 & & & \\
\hline \multicolumn{10}{|l|}{ Early Permian } \\
\hline $\mathrm{P}_{1}$ & 5 & 40.6 & 79.5 & 56.1 & 179.4 & 2.5 & & Fang et al., 1998 & FT \\
\hline $\mathrm{P}_{1}$ & 21 & 40.5 & 78.8 & 54.5 & 172.3 & 4.0 & & Sharps et al., 1989 & \\
\hline *Ave, 280 & $2 / 2$ & & & 55.4 & 175.8 & 9.5 & & & \\
\hline \multicolumn{10}{|l|}{ Late Permian } \\
\hline $\mathrm{P}_{2}$ & 7 & 44.0 & 88.1 & 75.8 & 195.0 & 13.6 & 18.5 & Nie et al., 1991 & $\mathrm{FT}, \mathrm{N}+\mathrm{R}$ \\
\hline $\mathrm{P}_{2}$ & 10 & 42.1 & 83.4 & 73.2 & 191.0 & 7.2 & 10.3 & McFadden et al., 1988 & $\mathrm{~N}+\mathrm{R}$ \\
\hline $\mathrm{P}_{3}$ & 21 & 40 & 79 & 65.6 & 181.2 & 3.9 & & Li et al., 1988, & $\mathrm{N}+\mathrm{R}$ \\
\hline $\mathrm{P}_{3}-\mathrm{T}_{1}$ & 16 & 42.1 & 83.3 & 71.8 & 187.6 & 5.5 & 7.8 & McFadden et al., 1988 & FT \\
\hline *Ave, 255 & $4 / 4$ & & & 71.2 & 186.7 & 4.0 & & & \\
\hline
\end{tabular}


Triassic

\begin{tabular}{|c|c|c|c|c|c|c|c|c|c|}
\hline $\mathrm{T}_{1}$ & 5 & 41 & 74.9 & 52.8 & 175.5 & 6.3 & 10.1 & Zhu et al., 1998 & $\mathrm{~N}+\mathrm{R}$ \\
\hline $\mathrm{T}_{2}$ & 26 & 40.9 & 81.5 & 52.5 & 168.2 & 4.2 & 6.1 & Zhu et al., 1998 & $\mathrm{~N}+\mathrm{R}$ \\
\hline $\mathrm{T}_{3}$ & 8 & 41.7 & 80.5 & 52.1 & 166.8 & 5.6 & 8.1 & Zhu et al., 1998 & $\mathrm{~N}+\mathrm{R}$ \\
\hline $\mathrm{T}$ & 6 & 41.6 & 83.5 & 59 & 160 & 13 & & Li et al., 1990 & FT, N+R \\
\hline *Ave, 230 & $4 / 4$ & & & 54.2 & 167.9 & 5.6 & & & \\
\hline \multicolumn{10}{|c|}{ Middle Jurassic } \\
\hline$* 170$ & 10 & 36 & 79.2 & 53.9 & 186.4 & 4.4 & 7.6 & Zhu et al., 1998 & $\mathrm{~N}+\mathrm{R}$ \\
\hline \multicolumn{10}{|c|}{ Late Jurassic } \\
\hline$* 155$ & 6 & 41.7 & 80.5 & 68.6 & 171.8 & 5.7 & 7.8 & Fang et al., 1998 & $\mathrm{~N}+\mathrm{R}$ \\
\hline \multicolumn{10}{|c|}{ Early Cretaceous } \\
\hline $\mathrm{J}_{3}-\mathrm{K}_{1}$ & 13 & 44.2 & 86.0 & 72.3 & 227.3 & 4.8 & 7.2 & Chen et al., 1991 & FT, N+R \\
\hline $\mathrm{J}_{3}-\mathrm{K}_{1}$ & 6 & 41.8 & 82.0 & 65.0 & 209.0 & 9 & 9 & Li et al., 1988 & $\mathrm{~N}+\mathrm{R}$ \\
\hline $\mathrm{K}_{1}$ & 7 & 39.5 & 75 & 66.3 & 226.6 & 9 & 15.9 & Chen et al., 1992 & $\mathrm{~N}+\mathrm{R}$ \\
\hline $\mathrm{K}_{1}$ & 3 & 38.5 & 76.4 & 70.4 & 212.1 & 6.6 & 10.8 & Chen et al., 1992 & $\mathrm{~N}+\mathrm{R}$ \\
\hline $\mathrm{K}_{1}$ & 8 & 36.3 & 78.8 & 72.3 & 206.6 & 9.8 & 15.9 & Zhu et al., 1998 & FT, N+R \\
\hline *Ave, 120 & $5 / 5$ & & & 69.5 & 216.3 & 4.6 & & & \\
\hline \multicolumn{10}{|c|}{ Late Cretaceous } \\
\hline $\mathrm{K}_{2}$ & 7 & 41.6 & 83.5 & 66.3 & 222.9 & 8.7 & 8.7 & Li et al., 1988 & FT \\
\hline $\mathrm{K}_{2}$ & 10 & 42 & 82.9 & 64 & 229 & 7.3 & 12.7 & Zhu et al., 1998 & $\mathrm{~N}+\mathrm{R}$ \\
\hline $\mathrm{K}_{2}$ & 11 & 39.5 & 75 & 70.8 & 222.6 & 5.4 & 8.9 & Chen et al., 1992 & FT \\
\hline $\mathrm{K}_{2}$ & 6 & 38.5 & 76.4 & 71 & 234 & 6.8 & 11.6 & Chen et al., 1992 & $\mathrm{~N}+\mathrm{R}$ \\
\hline $\mathrm{K}_{2}$-E & 9 & 44.2 & 86.0 & 74.3 & 223.1 & 6.4 & 9.4 & Chen et al., 1991 & $\mathrm{FT}, \mathrm{N}+\mathrm{R}$ \\
\hline *Ave, 80 & $5 / 5$ & & & 69.3 & 226.4 & 4.2 & & & \\
\hline \multicolumn{10}{|l|}{ Paleogene } \\
\hline$* 50$ & 4 & 41.6 & 83.5 & 73 & 245.4 & 6.0 & 9.7 & Fang et al., 1998 & \\
\hline \multicolumn{10}{|l|}{ Quaternary } \\
\hline$* 2$ & 3 & 36.2 & 81.5 & 79.9 & 183.1 & 1.6 & 2.4 & Meng et al., 1998 & \\
\hline
\end{tabular}

Poles with * were plotted in figure $\mathrm{S} 3$.

Abbreviations: S/N, the number of sites/drilled cores; Slong/Slat, longitude and latitude of sampling site; Plat/Plong, latitude and longitude of paleomagnetic pole; Dp/Dm, oval of $95 \%$ confidence for the paleomagnetic pole; A95, radius that mean direction lies within 95\% confidence. FT, fold test; CT, conglomerate test; N+R, existence of both normal and reversed polarities.

References:

Bai, Y.H., Chen, G.L., Sun, Q.G., Sun, Y.H., Li, Y.A., Dong, Y.J., Sun, D.J., 1987. Late Pale-ozoic polar wander path for the Tarim platform and its tectonic significance.Tectonophysics 139, 145-153.

Chen, Y., Cogné, J.P., Courtillot, V., 1992. New paleomagnetic poles from the TarimBasin, Northwestern China. Earth Planet. Sci. Lett. 114, 17-38.

Chen, Y., Cogné, J.P., Courtillot, V., Avouac, J.Ph., Tapponnier, P., Buffetaut, E., Wang, G.,Bai, M., You, H., Li, M., Wei, C., 1991. Paleomagneitc study of Mesozoic continentalsediments along the northern 
Tien Shan (China) and heterogeneous strain incentral Asia. J. Geophys. Res. 96, 4065-4082.

Chen, Y., Xu, B., Zhan, S., Li, Y.A., 2004. First mid-Neoproterozoic paleomagnetic results from the Tarim Basin (NW China) and their geodynamic implications. Precambrian Res.133, 271-281.

Fang, D.J., Jin, G.H., Jiang, L.P., Wang, P.Y., Wang, Z.L., 1996. Paleozoic paleomagneticresults and the tectonic significance of Tarim palte. Acta Geophys. Sin. 39 (4),522-532 (in Chinese with English abstract).

Fang, D.J., Wang, P.Y., Shen, Z.Y., Tan, X.D., 1998. Paleomagnetic results and Phanero-zoic apparent polar wandering path of Tarim block. Sci. China Ser. D 41 (Suppl.),105-112.

Huang, B.C., Xu, B., Zhang, C.X., Li, Y.A., Zhu, R.X., 2005. Paleomagnetism of the Baiy-isi volcanic rocks (ca. $740 \mathrm{Ma}$ ) of Tarim Northwest China: a continental fragment of Neoproterozoic Western Australia? Precambrian Res.142, 83-92.

Li, Y.P., McWilliams, M., Cox, A., Shrarps, R., Li, Y., Gao, Z., Zhang, Z., Zhai, Y., 1988. Late Permian Paleomagnetic pole from dikes of the Tarim craton, China. Geology16, 275-278.

Li, Y.P., McWilliams, M., Sharps, R., Li, Y.A., Gao, Z.J., 1990. A Devonian paleomagneticpole from red beds of the Tarim Block, China. J. Geophys. Res. 98, 185-198.

McFadden, P.L., Ma, X.H., McElhinny, M.W., Zhang, Z.K., 1988. Permo-Triassic mag-netostratigraphy in China: northern Tarim. Earth Planet. Sci. Lett. 87, 152-160.

Meng, Z.F., Deng, Y.S., Ding, Z.H., Zheng, Y.P., Li, Y.A., Sun, D.J., 1998. New paleo-magnetic results from Ceno-Mesozoic volcanic rocks along southern rim of theTarim Basin, China. Sci. China Ser. D 41 (Suppl.), 91-104.

Nie, S.Y., 1991. Paleoclimatic and paleomagnetic constraints on the Paleozoic recon-struction of south China, north China and Tarim. Tectonophysics 196, 279-308.

Sharps, R., McWilliams, M., Li, Y.P., Cox, A., Zhang, Z.K., Zhai, Y.J., Gao, Z.J., Li, Y.A.,Li, Q., 1989. Lower Permian paleomagnetism of the Tarim block, northwesternChina. Earth Planet. Sci. Lett. 92, 275-291.

Wen, B., Evans, D.A.D., and Li, Y.X., 2017, Neoproterozoic paleogeography of the Tarim Block: An extended or alternative "missing-link" model for Rodinia?: Earth and Planetary Science Letters, v. 458, p. 92-106, https:// doi.org/10.1016/j .eps1.2016.10.030.

Wen, B., Evans, D.A.D., Wang, C., Li, Y.X., and Jing, X., 2018, A positive test for the Greater Tarim Block at the heart of Rodinia: Mega-dextral suturing of supercontinent assembly: Geology, v. 46(8), p. 687-690, https://doi.org/10.1130/G40254.1

Wen, B., Li, Y.X., Zhu, W.B., 2013. Paleomagnetism of the Neoproterozoic diamictites of the Qiaoenbrak formation in the Aksu area, NW China: constraintson the paleogeographic position of the Tarim Block. Precambrian Res.226, 75-90.

Zhan, S., Chen, Y., Xu, B., Wang, B., Faure, M., 2007. Late Neoproterozoic paleomag-netic results from the Sugetbrak Formation of the Aksu area, Tarim basin (NW China) and their implications to paleogeographic reconstructions and the snow-ball Earth hypothesis. Precambrian Res.154, 143158.

Zhao, P., Chen, Y., Zhan, S., Xu, B., Faure, M., 2014. The apparent polar wander path of the Tarim block (NW China) since the Neoproterozoic and its implications for a long-term Tarim-Australia connection. Precambrian Res.242, 39-57.

Zhu, R.X., Yang, Z.Y., Wu, H.N., Ma, X.H., Huang, B.C., Meng, Z.F., Fang, D.J., 1998.Paleomagnetic apparent polar wander paths and movement for China maincontinental blocks during Phanerozoic. Sci. China Ser. D 28 (Suppl.), 1-16. 
Table S3. Compilation of 1000-800 Ma palaeomagnetic poles from main cratons of Rodinia. Abbreviations: Slat (Plat), latitude of sampling site (pole); Slong (Plong), longitude of sampling site (pole); A95, 95\% confidence limit; FT, fold test; CT, conglomerate test; BT, backed test; N+R, existence of both normal and reversed polarities.

\begin{tabular}{|c|c|c|c|c|c|c|c|c|c|}
\hline Pole ID & Age & location & Rock unit & $\begin{array}{l}\text { Plat } \\
\left({ }^{\circ} \mathrm{N}\right)\end{array}$ & $\begin{array}{l}\text { Plong } \\
\left({ }^{\circ} \mathrm{E}\right)\end{array}$ & $\begin{array}{c}\mathrm{A}_{95}\left(^{\circ}\right) \\
(\mathrm{dp} / \mathrm{dm})\end{array}$ & $\begin{array}{c}\text { paleolatitude } \\
\left({ }^{\circ} \mathrm{N}\right)\end{array}$ & Test & References \\
\hline \multicolumn{10}{|l|}{ Tarim } \\
\hline QV & $909-903$ & Aksu & Qigelekekuotan volcanics & -0.5 & 62.3 & 11.8 & $47.0 \pm 11.8$ & $\mathrm{FT}, \mathrm{N}+\mathrm{R}$ & This study \\
\hline SGV & $890-870$ & Yecheng & Sailajiazitage Group volcanics & -23.5 & 37.0 & 11.3 & $18.6 \pm 11.3$ & $\mathrm{CT}$ & Wen et al., 2018 \\
\hline \multicolumn{10}{|l|}{ Laurentia } \\
\hline L990 & 990 & USA & Adirondack fayalite granite & -28.4 & 132.7 & 6.9 & & $\mathrm{~N}+\mathrm{R}$ & Brown and McEnroe, 2012 \\
\hline L970 & 970 & USA & Adirondack anorthosites & -25.1 & 149.0 & 11.6 & & $\mathrm{~N}+\mathrm{R}$ & Brown and McEnroe, 2012 \\
\hline L960 & 960 & USA & Adirondack microcline gneiss & -18.4 & 151.1 & 10.5 & & $\mathrm{~N}+\mathrm{R}$ & Brown and McEnroe, 2012 \\
\hline L950 & 950 & USA & Magnetawan metaseds & -24.0 & 130.0 & & & & McWilliams and Dunlop, 1975 \\
\hline L900 & 900 & USA & Nankoweap Formation & -10.0 & 163.0 & $3.5 / 9.3$ & $-2.1 \pm 5.0$ & $\mathrm{~N}+\mathrm{R}$ & Weil et al., 2003 \\
\hline \multicolumn{10}{|l|}{ South China } \\
\hline SC824 & 824 & Yanbian & Mafic dykes & 45.1 & 130.4 & 19.0 & $57.9 \pm 20.6$ & & Niu et al., 2016 \\
\hline SC821 & 821 & Hubei & Xiaofeng dykes & 13.5 & 91.0 & $10.5 / 11.3$ & $64.5 \pm 10.9$ & & Li et al., 2004 \\
\hline SC805 & $805-802$ & Hunan & Madiyi Formation & 35.3 & 67.9 & $4.7 / 5.5$ & $53.8 \pm 4.8$ & $N+R$ & Xian et al., 2020 \\
\hline SC 800 & 800 & Yunnan & Chengjiang Formation & 32.8 & 56.3 & $7.1 / 8.6$ & $49.3 \pm 7.8$ & $\mathrm{~N}+\mathrm{R}$ & Jing et al., 2020 \\
\hline
\end{tabular}

Brown, L.L., and McEnroe, S.A., 2012, Paleomagnetism and magnetic mineralogy of Grenville metamorphic and igneous rocks, Adirondack Highlands, USA: Precambrian Research, v. 212-213, p. 57-74, https:// doi .org /10.1016/j .precamres .2012.04.012.

Jing, X., Yang, Z., Evans, D.A.D., Tong, Y., Xu, Y., and Wang, H., 2020, A pan-latitudinal Rodinia in the Tonian true polar wander frame: Earth and Planetary Science Letters, v. 530, p. 115880, https://doi .org/10.1016/ j.eps1.2019.115880.

Li, Z.X., Evans, D.A.D., and Zhang, S.H., 2004. A 90 spin on Rodinia: possible causal links between the Neoproterozoic supercontinent, superplume, true polar wander and lowlatitude glaciation. Earth Planet. Sci. Lett. 220 (3), 409-421. doi:10.1016/S0012-821X(04)00064-0

McWilliams, M.O., and Dunlop, D.J., 1975, Precambrian paleomagnetism: magnetizations reset by the Grenville orogeny: Science, v. 190, p. 269-272, DOI: 10.1126/science.190.4211.269

Niu, J., Li, Z.-X., and Zhu, W., 2016, Palaeomagnetism and geochronology of mid-Neoproterozoic Yanbian dykes, south China: Implications for a c. 820-800 Ma true polar wander event and the reconstruction of Rodinia, in Li, Z.-X., Evans, D.A.D., and Murphy, J.B., eds., Supercontinent Cycles Through Earth History: Geological Society [London] Special Publication 424, p. 191-211, https://doi .org/10.1144/SP424.11.

Weil, A.B., Geissman, J.W., Heizler, M., Van der Voo, R., 2003, Paleomagnetism of Middle Proterozoic mafic intrusions and Upper Proterozoic (Nankoweap) red beds from the Lower Grand Canyon Supergroup, Arizona: Tectonophysics, v. 375, p. 199-220, doi:10.1016/S0040-1951(03)00339-1

Wen, B., Evans, D.A.D., Wang, C., Li, Y.X., and Jing, X., 2018, A positive test for the Greater Tarim Block at the heart of Rodinia: Mega-dextral suturing of supercontinent assembly: Geology, v. 46(8), p. 687-690, https://doi.org/10.1130/G40254.1

Xian, H., Zhang, S., Li, H., Yang, T., and Wu, H., 2020, Geochronological and palaeomagnetic investigation of the Madiyi Formation, lower Banxi Group, South China: Implications for Rodinia reconstruction: Precambrian Research, v, 336, 105494, https://doi.org/10.1016/j.precamres.2019.105494 